\title{
Prognostic Markers in Peripheral T-Cell Lymphoma
}

\author{
Pier Paolo Piccaluga • Claudio Agostinelli • \\ Anna Gazzola • Claudia Mannu • Francesco Bacci • \\ Elena Sabattini • Stefano A. Pileri
}

Published online: 6 August 2010

(C) The Author(s) 2010. This article is published with open access at Springerlink.com

\begin{abstract}
Based on their own experience and knowledge of the literature, the authors review the pathobiological characteristics of peripheral T-cell lymphomas (PTCLs), focusing on the available prognostic indicators. The International Prognostic Index (IPI), which is based on age, performance status, lactate dehydrogenase [LDH], stage, and extranodal involvement, appears to be efficient as a prognostic index for PTCLs, at least in part and especially for certain PTCL subtypes. However, it is not so satisfactory for the two commonest PTCLs, PTCL not otherwise specified (PTCL/NOS) and angioimmunoblastic T-cell lymphoma (AITL), for which novel scores, possibly based on the biologic features of the tumors, have been explored. An Italian cooperative group proposed a revision of the IPI for PTCL unspecified (PTCL-U), the Prognostic Index for PTCL-U (PIT), which includes age, performance status, LDH, and bone marrow involvement. The PIT apparently offered some advantages, but they were not confirmed in subsequent studies. A clinical-biological score (the Bologna score) was then proposed, including tumor proliferation and clinical features (age, LDH, and performance status). This score appears promising and offers the intriguing advantage of integrating biological and clinical elements, but independent validation on a large series is still warranted. More recently, gene expression profiling has been used to identify novel molecular prognostic factors. In particular, inactivation of the NFKB pathway, high expression of proliferation-associated genes, and cyto-
\end{abstract}

P. P. Piccaluga $(\bowtie) \cdot$ C. Agostinelli $\cdot$ A. Gazzola $\cdot$ C. Mannu

F. Bacci $\cdot$ E. Sabattini $\cdot$ S. A. Pileri

Department of Hematology and Oncological Sciences "L. and A.

Seràgnoli”, Hematopathology Unit, S. Orsola-Malpighi Hospital,

University of Bologna,

Via Massarenti, 9,

40138 Bologna, Italy

e-mail: pierpaolo.piccaluga@unibo.it toxic molecular phenotype seem to be associated with a worse outcome. So far, however, none of these indicators has been validated in an independent series. Finally, various reports have dealt specifically with the prognostication of NK-derived tumors, including nasal and nasaltype lymphomas. Both the IPI and dedicated models have turned out to be of prognostic relevance for these tumors. In conclusion, although the IPI is somewhat effective for PTCL prognostication, novel scores that are more refined and possibly disease-specific are warranted. The validation process for several models, including clinical-pathological and molecular models, is now ongoing.

Keywords Peripheral T-cell lymphoma PTCL .

Prognostication - Gene expression profile .

International prognostic index $\cdot$ Bologna score

\section{Introduction}

In 1994, the Revised European-American Lymphoma (REAL) Classification introduced new standards in the lymphoma field [1]. In particular, it stated for the first time that a classification of lymphoid tumors should comprise a list of "real" entities, each defined by the amalgamation of cell morphology, phenotype, molecular genetics, clinical data, and identification of a normal counterpart, if possible [1]. After a validation trial [2], the REAL Classification was adopted by the World Health Organization (WHO) as guideline for lymphoma diagnosis and therapy, and its methodology was extended to all tumors of the hematopoietic system [3••]. Based on patients' survival without any treatment, non-Hodgkin lymphomas (NHLs) are classified as indolent (survival measurable in years) or aggressive (survival measurable in months). 
Peripheral T-cell lymphomas (PTCLs) belong to the aggressive lymphoma group [4••]. They represent approximately $12 \%$ of all lymphoid neoplasms $[4 \bullet \bullet, 5 \bullet \cdot$. Their incidence varies in different countries and races, being higher in HTLV-1 endemic areas (Asia, the Caribbean basin,

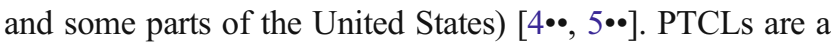
heterogeneous group of tumors that can be roughly subdivided into specified and not otherwise specified (NOS) forms $[5 \bullet \bullet, 6 \bullet$. The NOS forms, corresponding to about $60 \%$ to $70 \%$ of T-cell lymphomas, cannot be further classified on the basis of morphology, phenotype, and conventional molecular studies [4••]. Usually, they occur in the fifth or sixth decade of life, without sex predilection [710]. Although PTCLs/NOS can present as isolated disease, they more often have a widespread dissemination (stage IIIIV) with nodal, skin, liver, spleen, bone marrow, and peripheral blood involvement [7-10]. B symptoms are recorded in about $45 \%$ of cases at diagnosis. A hemophagocytic syndrome may also be encountered [7-10].

The tumor morphology is highly variable, comprising cells of different sizes and shapes [4••]. PTCLs/NOS may contain prominent reactive components, including small lymphocytes, eosinophils, plasma cells, histiocytes, and epithelioid elements $[4 \bullet \bullet]$.

Immunohistochemistry generally shows T-cellassociated molecular expression, but the phenotypic profile is aberrant in about $80 \%$ of cases [11].

Clonal rearrangements of T-cell receptor encoding genes are generally detected [12]. The karyotype is aberrant in more than $80 \%$ of patients and often is characterized by complex abnormalities. However, specific alterations have not been identified [13]. Recently, some recurrent lesions have been documented by comparative genomic hybridization and analysis of single-nucleotide polymorphisms (SNPs) $[14,15]$.

On clinical grounds, PTCLs/NOS are among the most aggressive NHLs. In most cases, the response to conventional chemotherapy is indeed frustrating, with rates of relapse-free and overall survival (OS) at 5 years less than $30 \%$ [5*•]. Notably, histologic classification remains a basic prognostic indicator $[5 \cdot \bullet, 6 \bullet, 8,16]$. Nodal and extranodal entities are clinically distinct, as extranodal tumors, especially the cutaneous forms, often have a relatively good outcome [5••]. In addition, among nodal PTCLs, the distinction between anaplastic large-cell lymphoma (ALCL) and other entities retains a significant prognostic impact, as does the distinction between anaplastic lymphoma kinase-positive $\left(\mathrm{ALK}^{+}\right)$and $\mathrm{ALK}^{-}$cases among ALCLs, $[5 \bullet \bullet, 6 \bullet, 8,16]$. In fact, patients with $\mathrm{ALK}^{+}$ALCL, particularly children and young adults, have a significantly better clinical outcome than patients with all other forms $[5 \bullet \bullet, 6 \bullet]$. Importantly, it was recently suggested that $\mathrm{ALK}^{-}$ ALCL should be included in PTCLs/NOS, based on a lack of evidence of clear biologic differences between them, but new clinical and molecular findings have demonstrated that $\mathrm{ALK}^{-}$ALCL and PTCL/NOS are distinct entities, also presenting with different clinical outcomes $[6 \bullet, 17 \bullet \bullet, 18]$.

In addition to the basic distinction of the different entities, several attempts have been made in recent years to offer reliable prognostic indicators. Clinical, pathological, clinical-pathological, and molecular scores have been proposed and are briefly discussed in this article (Table 1).

\section{International Prognostic Index (IPI)}

The International Prognostic Index (IPI) was first introduced in 1993 with the intent of identifying patients affected by aggressive NHL, with its greater risk of treatment failure, relapse, and death [19]. It is based on clinical parameters, such as tumor stage, extranodal localization, age, lactate dehydrogenase (LDH) level, and performance status. It was largely based on B-NHL rather than T-NHL, especially diffuse large B-cell lymphomas (DLBCLs), but its ability to stratify PTCL patients was reported in later years. In particular, the International Lymphoma Study Group showed that OS and relapse-free survival were significantly different in patients with low IPI (0/1) versus high (4/5) IPI for all PTCL types except ALCL (5-year OS, $36 \%$ vs $15 \%$; 5 -year failure-free survival, $27 \%$ vs $10 \%$ ) [2]. On the other hand, subsequent studies showed that the IPI was particularly effective for prognostication in both $\mathrm{ALK}^{+}$and $\mathrm{ALK}^{-}$ALCL $[6 \cdot, 20]$. In particular, in $\mathrm{ALK}^{+} \mathrm{ALCL}$, the 5 -year OS was $94 \% \pm 5 \%$ for the low $/$ low-intermediate risk group versus $41 \% \pm 12 \%$ for the high/high-intermediate group $(P<0.0001)$ [20]. In fact, the IPI was more relevant than ALK expression in stratifying patients with ALCL: the relative risk of death was 3.50 for the high/high-intermediate IPI group versus 0.29 for the low/low-intermediate group, though both the IPI score and ALK expression were significant prognostic factors [20]. In addition, in a large study within the International T-cell Lymphoma Project (ITCLP), the IPI effectively identified risk groups with different prognoses within both $\mathrm{ALK}^{+}$ and $\mathrm{ALK}^{-}$ALCL, although those with an IPI score of 3 or more fell into the poor-risk category regardless of ALK status $[6 \bullet]$.

Furthermore, Suzumiya et al. [21•] recently reported on a large series of patients with aggressive adult T-cell leukemia/lymphoma (ATLL), providing evidence that IPI, platelet count, and B symptoms were significant prognostic factors. Interestingly, multivariate analysis indicated that only the IPI was an independent predictor of OS in this series, though the IPI prediction of OS was significant only for the lymphoma type of ATLL $(P=0.04)$, not for the acute type $(P=0.24)[21 \bullet]$. 
Table 1 Prognostic markers and scores in peripheral T-cell lymphomas (PTCLs)

\begin{tabular}{lll}
\hline Prognostic indicator & PTCL subtype & Studies \\
\hline Histotype & All & The Non-Hodgkin's Lymphoma Classification Project [2]; Vose et al. [5••]; \\
International Prognostic & All & $\begin{array}{l}\text { Savage et al. [6•]; Lopez-Guillermo et al. [8]; Ascani et al. [16] } \\
\text { The Non-Hodgkin's Lymphoma Classification Project [2] }\end{array}$ \\
& ALCL & Savage et al. [6•]; Falini et al. [20] \\
& ATLL & Suzumiya et al. [21•] \\
NK/T-cell lymphoma & Au et al [57•] \\
Bologna score & PTCL/NOS & Gallamini et al. [22]; Savage et al. [6] \\
Korean prognostic index & PTCL/NOS, AITL & Went et al. [11]; Briones et al. [34] \\
NK prognostic index & NK/T-cell lymphoma & Lee et al. [54] \\
EBV & STCL/NOS, AITL & Went et al. [11]; Kluin et al. [24]; Dupuis et al. [25] \\
& NK/T-cell lymphoma & Au et al. [62]; Cheung et al. [60]; Chim et al. [64]; Huang et al. [65]; Lee et al. [66]; \\
& Ng et al. [63] \\
Proliferation index & PTCL/NOS & \\
Ki-67 & & Went et al. [11] \\
Molecular signature & & Cuadros et al. [43•] \\
Cellular derivation & PTCL/NOS & Went et al. [11]; Bekkenk et al. [26]; Kojima et al. [27]; Iqbal et al. [48•] \\
NFkB activation & PTCL/NOS & Martinez-Delgado et al. [36]; Ballester et al. [38]; Briones et al. [34] \\
CYP3A & PTCL/NOS & Rodríguez-Antona et al. [49] \\
\hline
\end{tabular}

AITL angioimmunoblastic T-cell lymphoma; $A L C L$ anaplastic large-cell lymphoma; $A T L L$ adult T-cell leukemia/lymphoma; $N K$ natural killer; $P T C L / N O S$ peripheral T-cell lymphoma not otherwise specified

${ }^{\text {a }}$ Prognostic impact not statistically significant

Overall, IPI identification of patients at higher risk is the expression of disease extension and the patient's frailty. It offers neither specific biologic hints nor potential targets for overcoming drug resistance. It soon became clear that the IPI was not as effective in PTCLs/NOS and angioimmunoblastic T-cell lymphomas (AITLs) (the two commonest PTCL types) as in the original series of DLBCLs, probably reflecting, at least in part, the fact that PTCL therapy is basically derived from B-NHLs and specific trials have been lacking for a long time. Thus, novel prognostic scores for PTCLs have been investigated in the past few years.

\section{Prognostic Index for PTCL/NOS}

In 2004, an Italian group (Intergruppo Italiano Linfomi) proposed a novel prognostic model based on a retrospective, multicentric clinical analysis of 385 patients with PTCL unspecified (PTCL-U) [22]. The new model, the Prognostic Index for PTCL-U (PIT), included bone marrow involvement, age, performance status, and LDH. When these four variables were combined in four groups, the PIT could identify patient subgroups with different outcomes. The PIT turned out to be slightly more effective than the IPI in stratifying PTCL patients (log-rank 66.79 vs 55.94) and was then proposed as reference tool. In addition, a simplified, two-class PIT appeared to be superior to a simplified two-class IPI (log-rank 49.36 vs 30.23) [22]. However, the PIT was based on a series lacking systematic histologic review and, although its value was confirmed within the International Peripheral T-Cell Lymphoma Project (ITCLP), its results were not superior to those of the IPI. In particular, the PIT was also applied to $\mathrm{ALK}^{+}$and $\mathrm{ALK}^{-} \mathrm{ALCL}$ and was similarly predictive of failure-free survival and OS in both groups [6॰]. However, given that the distribution of patients across the risk groups was very similar with the two prognostic models and that bone marrow involvement is rarely observed in ALCL, the PIT actually seems to mirror the IPI in this setting [6•]. In addition, as it does not include tumor-specific biologic factors, cannot be intended for the future application of targeted therapies.

\section{Clinical-Pathologic Prognostic Score (Bologna Score)}

Immunohistochemical markers have been largely proposed for prognostication of malignant lymphomas. In a large collection of Italian PTCL cases, Went et al. [11] found that high Ki-67 expression, positive Epstein-Barr virus (EBV) status, and CD15 staining were associated with the worst outcome in PTCL/NOS. Interestingly, EBV has repeatedly 
been proposed as a negative prognosticator in PTCLs [23], among both Asian and European patients [24, 25]. Specifically, Went et al. [11] found EBV positivity in 5\% of patients with PTCL/NOS and $3 \%$ of those with AITL, values definitely lower than the value recorded by Dupuis et al. [25] in a French PTCL cohort. Such discrepancies may reflect geographic or racial differences. No other immunohistochemical marker alone or in combination was associated with a poor outcome, although patients with tumors expressing $\mathrm{CD} 57$ or $\mathrm{CD} 4^{+} / \mathrm{CD} 8^{-}$phenotype showed a tendency for a better outcome; the possible prognostic relevance of the latter phenotype has also been proposed by others [26, 27].

Furthermore, based on a large series provided with follow-up data, Went et al. [11] developed a new score integrating both patient-specific and tumor-specific characteristics (age $>60$ years, performance status, $\mathrm{LDH}$, and Ki67 protein $\geq 80 \%$ ) and identifying three clearcut groups of patients with different responses to therapy and differing life expectancy (median OS, 37 vs 23 vs 6 months; $P<0.001$ ) [11, 22, 28-33]. This score, the Bologna score, seemed to be better able to predict patient outcome than previous indices, including the IPI $(P<0.001$ vs 0.1$)$ and PIT $(P<0.001$ vs 0.0043$)$. The prognostic ability of the Bologna score was recently validated by a Spanish group [34]. Remarkably, all the factors contributing to the scoring system proposed by Went et al. [11], which incorporates both patient-specific and tumor-specific characteristics, are part of the routine workup, making their integration simple and cost-effective.

\section{Gene Expression Profiling}

In the past few years, numerous studies have dealt with the gene expression profiling (GEP) of nodal PTCLs [17••, 18, 35-38, 39••, 40••, 41, 42, 43•, 44-47, 48•], possibly providing novel insight into PTCL prognostication. First, a few reports suggested that PTCLs/NOS may present with upregulation or downregulation of NFKB molecules [35, $36,38]$, with possible prognostic relevance [36, 38]. In particular, cases with lower levels of NFKB-related molecules or other evidence of NFKB inactivity showed better survival, with a median OS of 25 months (range 0 124 months) versus a median OS of 12 months (range $0-19$ months $)(P=0.032)[36,38]$. This observation was confirmed by another Spanish group, which demonstrated 5 -year OS of $45 \%$ in $\mathrm{NFKB}^{+}$cases versus $0 \%$ in $\mathrm{NFKB}^{-}$ cases $(P=0.04)$ [34]. However, all these studies included a relatively limited number of cases, mixed different histotypes $[34,36]$, or included patients with prominent nonneoplastic components [38], which may have influenced the results, at least in part.
In addition, based on GEP obtained from 35 patients with nodal PTCL (23 with PTCL/NOS and 12 with AITL), it was suggested that overexpression of genes involved in a "proliferation signature" was associated significantly with shorter survival [43॰]. This proliferation signature included genes commonly associated with the cell cycle, such as $C C N A, C C N B, T O P 2 A$, and PCNA [43•]. Notably, this evidence of high proliferation as a possible adverse prognostic factor was definitely in line with the findings of Went et al. [11] and the ITCLP (unpublished), highlighting the importance of this parameter.

Finally, based on GEP analyses, our group indicated that PTCLs/NOS can be subclassified on the basis of histogenesis. In particular, at least two subgroups were described, derived from activated helper elements and cytotoxic elements $[17 \bullet \bullet]$. This finding was recently confirmed by Iqbal et al. [48•]. Intriguingly, this report also suggested that the cytotoxic profile may be associated with unfavorable outcome, though this evidence was based on a limited series and warrants further validation. On the other hand, the possibility of more favorable outcome for PTCL cases with a helper phenotype has been previously suggested by others [11, 26, 27].

Overall, GEP studies have provided evidence that molecular features may be useful in defining the prognosis of PTCL patients. However, no complete explanation has been offered regarding the molecular basis of drug resistance. Our group described for the first time the expression of molecules associated with drug resistance in solid tumors, such as CYR61 and NNMT in PTCL/NOS [17••]. Furthermore, Rodríguez-Antona et al. [49] recently found that a high expression of cytochrome P450 3A (CYP3A), an enzyme involved in the inactivation of chemotherapy drugs, was associated with poor response to standard PTCL chemotherapy, suggesting that CYP3A could be useful as a predictor of response. Indeed, the molecular classification of PTCLs and the identification of key events in their molecular pathology is likely to be the basis for future prognostication and targeted treatment in this field, as has occurred in DLBCL $[50,51 \bullet \bullet]$.

\section{Prognostication of NK/T-Cell Lymphoma, Nasal Type}

Extranodal natural killer (NK)/T-cell lymphoma, nasal type is a distinct entity in the WHO classification of lymphoid tumors; it is more frequent in Asia and in Central and South America than in Western countries [52-56, 57•]. Morphologically, tissue invasion, vascular destruction, and necrosis are the most prominent features; EBV is always integrated in the genome of neoplastic cells [56]. Most cases derive from NK cells and are characterized by a typical NK phenotype and T-cell receptor genes in germ-line configuration, but in some instances a cytotoxic T-lymphocyte 
origin has been recognized [56]. The nasal cavity and the upper aerodigestive tract (nasal NK/T-cell lymphoma) are the most commonly involved sites, but skin, the gastrointestinal tract, lung, testis, and soft tissues (extranasal NK/ T-cell lymphoma) can be also affected [52, 53, 56, 58].

The prognosis of extranodal NK/T-cell lymphoma is poor, the worst among the PTCL categories [57•]. The survival rate is $30 \%$ to $40 \%$, with some differences between nasal and nonnasal disease; the nonnasal disease is more aggressive $[56,57 \cdot, 59]$. The inclusion of radiotherapy in treatment protocols has improved the outcome of nasal NK/T-cell lymphoma in stage I or II $[55,56,57 \cdot, 60,61]$. Among nasal forms, adverse prognostic factors are unfavorable IPI, advanced-stage disease (stage III or IV), high circulating EBV DNA levels, and detection of EBV in bone marrow cells by in situ hybridization $[56,57 \bullet, 60$, 62-66]. Some studies suggest that a high proportion of large/transformed cells in the tumoral population has a negative impact on survival, but the significance of cytologic features as a prognostic indicator is still uncertain $[55,56,57 \cdot, 58]$. Primary extranasal cases are highly aggressive and the response to therapy is poor even in patients with localized disease [56, 57•].

A Korean group has proposed a new prognostic index specifically developed for NK/T-cell tumors, based on four parameters: B symptoms, LDH levels, stage, and regional lymph node involvement. This index demonstrated better prognostic stratification of NK/T-cell lymphomas than the IPI [54].

In addition, a recent study showed that four factors (nonnasal type, stage, performance status, and extranodal involvement) were significant prognostic factors in NK/Tcell lymphomas [67]. Using these four variables, an NK prognostic index was successfully constructed in which the 4 -year OS was $55 \%$ for patients with no adverse factors, $33 \%$ with one adverse factor, $15 \%$ with three factors, and $6 \%$ with four factors [67].

\section{Conclusions}

PTCLs have long represented an orphan pathology, as explained by their relatively low incidence (though higher than the incidence of a "common" tumor such as Hodgkin's lymphoma), the difficulties encountered in their analysis, and their dismal prognosis. During the past few years, however, a great deal of interest has developed and new light has been shed on the pathobiology of these tumors, and leading to the proposal of more effective prognosticators. Although the IPI is somewhat effective for PTCL prognostication, more refined and possibly disease-specific novel scores have been explored. Several models including clinical-pathological and molecular features have been proposed and are now being validated. In addition, innovative therapeutic schedules have been proposed on the basis of newly developed microarray techniques. We seem close to the morning of a new era in which the shadows that have wrapped PTCLs for several decades will be dissipated.

Acknowledgments This work was supported by Centro Interdipartimentale per la Ricerca sul Cancro "G. Prodi", BolognAIL, AIRC (IG4987), RFO (Prof. Pileri, Dr. Piccaluga); and Fondazione Cassa di Risparmio in Bologna, Fondazione della Banca del Monte e Ravenna, Progetto Strategico di Ateneo 2006 (Prof. Pileri and Dr. Piccaluga).

Disclosure No potential conflicts of interest relevant to this article were reported.

Open Access This article is distributed under the terms of the Creative Commons Attribution Noncommercial License which permits any noncommercial use, distribution, and reproduction in any medium, provided the original author(s) and source are credited.

\section{References}

Papers of particular interest, published recently have been highligted as:

- Of importance

•• Of major importance

1. Harris NL, Jaffe ES, Stein H, et al.: A revised European-American classification of lymphoid neoplasms: a proposal from the International Lymphoma Study Group. Blood 1994, 84(5):1361-1392.

2. A clinical evaluation of the International Lymphoma Study Group classification of non-Hodgkin's lymphoma. The Non-Hodgkin's Lymphoma Classification Project. Blood 1997, 89(11):3909-3918.

3. •- Swerdlow S, Campo E, Harris NL, et al. (eds): WHO Classification of Tumors of Hematopoietic and Lymphoid Tissues, edn 4. Lyon: IARC; 2008. This is the most recent update of the WHO classification of tumors of hematopoietic and lymphoid tissues.

4. • Pileri S, Ralfkiaer E, Weisenburger D, et al.: Peripheral T-cell lymphoma, not otherwise specified. In WHO Classification of Tumors of Hematopoietic and Lymphoid Tissues, edn 4. Edited by Swerdlow S, Campo E, Harris NL, et al. Lyon: IARC; 2008:429. This is the original chapter on PTCL/NOS published in the WHO Classification.

5. •• Vose J, Armitage J, Weisenburger D: International peripheral Tcell and natural killer/T-cell lymphoma study: pathology findings and clinical outcomes. J Clin Oncol 2008, 26(25):4124-4130. In this report, the authors describe some major findings within the International Peripheral T-Cell Lymphoma Project.

6. - Savage KJ, Harris NL, Vose JM, et al.: ALK- anaplastic largecell lymphoma is clinically and immunophenotypically different from both ALK+ ALCL and peripheral T-cell lymphoma, not otherwise specified: report from the International Peripheral TCell Lymphoma Project. Blood 2008, 111(12):5496-5504. In this report, the authors describe some major findings within the International Peripheral T-Cell Lymphoma Project concerning ALCL.

7. Evens AM, Gartenhaus RB: Treatment of T-cell non-Hodgkin's lymphoma. Curr Treat Options Oncol 2004, 5(4):289-303. 
8. Lopez-Guillermo A, Cid J, Salar A, et al.: Peripheral T-cell lymphomas: initial features, natural history, and prognostic factors in a series of 174 patients diagnosed according to the R.E.A.L. Classification. Ann Oncol 1998, 9(8):849-855.

9. Gisselbrecht C, Gaulard P, Lepage E, et al.: Prognostic significance of T-cell phenotype in aggressive non-Hodgkin's lymphomas. Groupe d'Etudes des Lymphomes de l'Adulte (GELA). Blood 1998, 92(1):76-82.

10. Effect of age on the characteristics and clinical behavior of nonHodgkin's lymphoma patients. The Non-Hodgkin's Lymphoma Classification Project. Ann Oncol 1997, 8(10):973-978.

11. Went P, Agostinelli C, Gallamini A, et al.: Marker expression in peripheral T-cell lymphoma: a proposed clinical-pathologic prognostic score. J Clin Oncol 2006, 24(16):2472-2479.

12. Rudiger T, Weisenburger DD, Anderson JR, et al.: Peripheral Tcell lymphoma (excluding anaplastic large-cell lymphoma): results from the Non-Hodgkin's Lymphoma Classification Project. Ann Oncol 2002, 13(1):140-149.

13. Zettl A, Rudiger T, Konrad MA, et al.: Genomic profiling of peripheral T-cell lymphoma, unspecified, and anaplastic large Tcell lymphoma delineates novel recurrent chromosomal alterations. Am J Pathol 2004, 164(5):1837-1848.

14. Oshiro A, Tagawa H, Ohshima K, et al.: Identification of subtypespecific genomic alterations in aggressive adult T-cell leukemia/ lymphoma. Blood 2006, 107(11):4500-4507.

15. Hartmann S, Gesk S, Scholtysik R, et al.: High resolution SNP array genomic profiling of peripheral $\mathrm{T}$ cell lymphomas, not otherwise specified, identifies a subgroup with chromosomal aberrations affecting the REL locus. Br J Haematol 2010, 148 (3):402-412.

16. Ascani S, Zinzani PL, Gherlinzoni F, et al.: Peripheral T-cell lymphomas. Clinico-pathologic study of 168 cases diagnosed according to the R.E.A.L. Classification. Ann Oncol 1997, 8(6):583-592.

17. •• Piccaluga PP, Agostinelli C, Califano A, et al.: Gene expression analysis of peripheral $\mathrm{T}$ cell lymphoma, unspecified, reveals distinct profiles and new potential therapeutic targets. J Clin Invest 2007, 117(3):823-834. In this article, the authors presented for the first time a global GEP study in the field of PTCLs, providing new evidence on their histogenesis and pathogenesis and proposing new therapeutic targets.

18. Piva R, Agnelli L, Pellegrino E, et al.: Gene expression profiling uncovers molecular classifiers for the recognition of anaplastic large-cell lymphoma within peripheral T-cell neoplasms. J Clin Oncol 2010, 28(9):1583-1590.

19. A predictive model for aggressive non-Hodgkin's lymphoma. The International Non-Hodgkin's Lymphoma Prognostic Factors Project. N Engl J Med 1993, 329(14):987-994.

20. Falini B, Pileri S, Zinzani PL, et al.: ALK+ lymphoma: clinicopathological findings and outcome. Blood 1999, 93(8):2697-2706.

21. - Suzumiya J, Ohshima K, Tamura K, et al.: The International Prognostic Index predicts outcome in aggressive adult T-cell leukemia/lymphoma: analysis of 126 patients from the International Peripheral T-Cell Lymphoma Project. Ann Oncol 2009, 20 (4):715-721. This is a large study within the International Peripheral T-Cell Lymphoma Project on aggressive adult T-cell leukemia/lymphoma.

22. Gallamini A, Stelitano C, Calvi R, et al.: Peripheral T-cell lymphoma unspecified (PTCL-U): a new prognostic model from a retrospective multicentric clinical study. Blood 2004, 103 (7):2474-2479

23. Cheng AL, Su IJ, Chen YC, et al.: Characteristic clinicopathologic features of Epstein-Barr virus-associated peripheral T-cell lymphoma. Cancer 1993, 72(3):909-916.

24. Kluin PM, Feller A, Gaulard P, et al.: Peripheral T/NK-cell lymphoma: a report of the IXth Workshop of the European Association for Haematopathology. Histopathology 2001, 38(3):250-270.
25. Dupuis J, Emile JF, Mounier N, et al.: Prognostic significance of Epstein-Barr virus in nodal peripheral T-cell lymphoma, unspecified: A Groupe d'Etude des Lymphomes de l'Adulte (GELA) study. Blood 2006, 108(13):4163-4169.

26. Bekkenk MW, Vermeer MH, Jansen PM, et al.: Peripheral T-cell lymphomas unspecified presenting in the skin: analysis of prognostic factors in a group of 82 patients. Blood 2003, 102(6):2213-2219.

27. Kojima H, Hasegawa Y, Suzukawa K, et al.: Clinicopathological features and prognostic factors of Japanese patients with "peripheral T-cell lymphoma, unspecified" diagnosed according to the WHO classification. Leuk Res 2004, 28(12):1287-1292.

28. Caulet-Maugendre S, Patey M, Granier E, et al.: Quantitative analysis of cellular proliferative activity in 35 T-cell nonHodgkin's lymphomas. Use of proliferating cell nuclear antigen and $\mathrm{Ki}-67$ (MIB-1) antibodies and nucleolar organizer regions. Anal Quant Cytol Histol 1996, 18(5):337-344.

29. Miller TP, Grogan TM, Dahlberg S, et al.: Prognostic significance of the Ki-67-associated proliferative antigen in aggressive nonHodgkin's lymphomas: a prospective Southwest Oncology Group trial. Blood 1994, 83(6):1460-1466.

30. Mochen C, Giardini R, Costa A, Silvestrini R: MIB-1 and S-phase cell fraction predict survival in non-Hodgkin's lymphomas. Cell Prolif 1997, 30(1):37-47.

31. Montalban C, Obeso G, Gallego A, et al.: Peripheral T-cell lymphoma: a clinicopathological study of 41 cases and evaluation of the prognostic significance of the updated Kiel classification. Histopathology 1993, 22(4):303-310.

32. Sheval EV, Churakova JV, Dudnik OA, Vorobjev IA: Examination of the proliferative activity of tumor cells in human lymphoid neoplasms using a morphometric approach. Cancer 2004, 102 (3):174-185.

33. Tiemann M, Schrader C, Klapper W, et al.: Histopathology, cell proliferation indices and clinical outcome in 304 patients with mantle cell lymphoma (MCL): a clinicopathological study from the European MCL Network. Br J Haematol 2005, 131(1):29-38.

34. Briones J, Moga E, Espinosa I, et al.: Bcl-10 protein highly correlates with the expression of phosphorylated p65 NF-kappaB in peripheral T-cell lymphomas and is associated with clinical outcome. Histopathology 2009, 54(4):478-485.

35. Martinez-Delgado B, Melendez B, Cuadros M, et al.: Expression profiling of T-cell lymphomas differentiates peripheral and lymphoblastic lymphomas and defines survival related genes. Clin Cancer Res 2004, 10(15):4971-4982.

36. Martinez-Delgado B, Cuadros M, Honrado E, et al.: Differential expression of NF-kappaB pathway genes among peripheral T-cell lymphomas. Leukemia 2005, 19(12):2254-2263.

37. Piccaluga PP, Agostinelli C, Zinzani PL, et al.: Expression of platelet-derived growth factor receptor alpha in peripheral T-cell lymphoma not otherwise specified. Lancet Oncol 2005, 6(6):440.

38. Ballester B, Ramuz O, Gisselbrecht C, et al.: Gene expression profiling identifies molecular subgroups among nodal peripheral T-cell lymphomas. Oncogene 2006, 25(10):1560-1570.

39. • de Leval L, Rickman DS, Thielen C, et al.: The gene expression profile of nodal peripheral T-cell lymphoma demonstrates a molecular link between angioimmunoblastic T-cell lymphoma (AITL) and follicular helper T (TFH) cells. Blood 2007, 109 (11):4952-4963. In this article, the authors provided molecular evidence that AITLs and some PTCLS/NOS derive from follicular T-helper cells.

40. •• Piccaluga PP, Agostinelli C, Califano A, et al.: Gene expression analysis of angioimmunoblastic lymphoma indicates derivation from $\mathrm{T}$ follicular helper cells and vascular endothelial growth factor deregulation. Cancer Res 2007, 67(22):10703-10710. In this article, the authors provided molecular evidence of AITL derivation from follicular T-helper cells and showed the aberrant expression of VEGF and VEGFR2 in such tumors. 
41. Piccaluga PP, Agostinelli C, Righi S, et al.: Expression of CD52 in peripheral T-cell lymphoma. Haematologica 2007, 92(4):566567.

42. Lamant L, de Reynies A, Duplantier MM, et al.: Gene-expression profiling of systemic anaplastic large-cell lymphoma reveals differences based on ALK status and two distinct morphologic ALK+ subtypes. Blood 2007, 109(5):2156-2164.

43. - Cuadros M, Dave SS, Jaffe ES, et al.: Identification of a proliferation signature related to survival in nodal peripheral T-cell lymphomas. J Clin Oncol 2007, 25(22):3321-3329. In this article, the authors provided evidence of the prognostic relevance of proliferation rate in PTCL/NOS, based on gene expression data.

44. Miyazaki K, Yamaguchi $\mathrm{M}$, Imai $\mathrm{H}$, et al.: Gene expression profiling of peripheral T-cell lymphoma including gammadelta $\mathrm{T}$ cell lymphoma. Blood 2009, 113(5):1071-1074.

45. Pise-Masison CA, Radonovich M, Dohoney K, et al:: Gene expression profiling of ATL patients: compilation of diseaserelated genes and evidence for TCF4 involvement in BIRC5 gene expression and cell viability. Blood 2009, 113(17):4016-4026.

46. Gazzola A, Bertuzzi C, Agostinelli C, et al.: Physiological PTEN expression in peripheral T-cell lymphoma not otherwise specified. Haematologica 2009, 94(7):1036-1037.

47. Huang Y, de Reynies A, de Leval L, et al.: Gene expression profiling identifies emerging oncogenic pathways operating in extranodal NK/ T-cell lymphoma, nasal-type. Blood 2010, 115(6):1226-1237.

48. - Iqbal J, Weisenburger DD, Greiner TC, et al.: Molecular signatures to improve diagnosis in peripheral T-cell lymphoma and prognostication in angioimmunoblastic T-cell lymphoma. Blood 2010, 115:919-920. This large study basically confirmed previous data and supported the idea that PTCL/NOS cases with different cellular derivation may have different clinical outcomes.

49. Rodriguez-Antona C, Leskela S, Zajac M, et al.: Expression of CYP3A4 as a predictor of response to chemotherapy in peripheral T-cell lymphomas. Blood 2007, 110(9):3345-3351.

50. Agostinelli C, Piccaluga PP, Went $\mathrm{P}$, et al.: Peripheral $\mathrm{T}$ cell lymphoma, not otherwise specified: the stuff of genes, dreams and therapies. J Clin Pathol 2008, 61(11):1160-1167.

51. • Lenz G, Staudt LM: Aggressive lymphomas. N Engl J Med 2010, 362(15):1417-1429. This is a comprehensive review on the molecular pathogenesis of aggressive B-NHL.

52. Quintanilla-Martinez L, Franklin JL, Guerrero I, et al.: Histological and immunophenotypic profile of nasal NK/T cell lymphomas from Peru: high prevalence of p53 overexpression. Hum Pathol 1999, 30(7):849-855.

53. Oshimi K, Kawa K, Nakamura S, et al.: NK-cell neoplasms in Japan. Hematology 2005, 10(3):237-245.

54. Lee J, Suh C, Park YH, et al.: Extranodal natural killer T-cell lymphoma, nasal-type: a prognostic model from a retrospective multicenter study. J Clin Oncol 2006, 24(4):612-618.
55. Barrionuevo C, Zaharia M, Martinez MT, et al.: Extranodal NK/Tcell lymphoma, nasal type: study of clinicopathologic and prognosis factors in a series of 78 cases from Peru. Appl Immunohistochem Mol Morphol 2007, 15(1):38-44.

56. Chan J, Quintanilla-Martinez L, Ferry J, Peh SC: Extranodal NK/ T-cell lymphoma, nasal-type. In WHO Classification of Tumors of Hematopoietic and Lymphoid Tissues, edn 4. Edited by Swerdlow S, Campo E, Harris NL, et al. Lyon: IARC; 2008:285.

57. - Au WY, Weisenburger DD, Intragumtornchai $\mathrm{T}$, et al.: Clinical differences between nasal and extranasal natural killer/T-cell lymphoma: a study of 136 cases from the International Peripheral T-Cell Lymphoma Project. Blood 2009, 113(17):3931-3937. This large study within the International Peripheral T-Cell Lymphoma Project concerns nasal and extranasal NK/T-cell lymphomas.

58. Kwong YL, Chan AC, Liang R, et al.: CD56+ NK lymphomas: clinicopathological features and prognosis. Br J Haematol 1997, 97(4):821-829.

59. Chan JK: Natural killer cell neoplasms. Anat Pathol 1998, 3:77-145.

60. Cheung MM, Chan JK, Lau WH, et al.: Early stage nasal NK/Tcell lymphoma: clinical outcome, prognostic factors, and the effect of treatment modality. Int J Radiat Oncol Biol Phys 2002, 54(1):182-190.

61. Kuo TT, Shih LY, Tsang NM: Nasal NK/T cell lymphoma in Taiwan: a clinicopathologic study of 22 cases, with analysis of histologic subtypes, Epstein-Barr virus LMP-1 gene association, and treatment modalities. Int J Surg Pathol 2004, 12(4):375-387.

62. Au WY, Pang A, Choy C, et al.: Quantification of circulating Epstein-Barr virus (EBV) DNA in the diagnosis and monitoring of natural killer cell and EBV-positive lymphomas in immunocompetent patients. Blood 2004, 104(1):243-249.

63. Ng SB, Lai KW, Murugaya S, et al.: Nasal-type extranodal natural killer/T-cell lymphomas: a clinicopathologic and genotypic study of 42 cases in Singapore. Mod Pathol 2004, 17(9):1097-1107.

64. Chim CS, Ma SY, Au WY, et al.: Primary nasal natural killer cell lymphoma: long-term treatment outcome and relationship with the International Prognostic Index. Blood 2004, 103(1):216-221.

65. Huang WT, Chang KC, Huang GC, et al.: Bone marrow that is positive for Epstein-Barr virus encoded RNA-1 by in situ hybridization is related with a poor prognosis in patients with extranodal natural killer/T-cell lymphoma, nasal type. Haematologica 2005, 90(8):1063-1069.

66. Lee J, Suh C, Huh J, et al.: Effect of positive bone marrow EBV in situ hybridization in staging and survival of localized extranodal natural killer/T-cell lymphoma, nasal-type. Clin Cancer Res 2007, 13(11):3250-3254.

67. Suzuki R, Suzumiya J, Yamaguchi M, et al.: Prognostic factors for mature natural killer $(\mathrm{NK})$ cell neoplasms: aggressive $\mathrm{NK}$ cell leukemia and extranodal NK cell lymphoma, nasal type. Ann Oncol 2010, 21(5):1032-1040. 